\title{
Acinetobacter baumannii and MRSA Contamination on Reusable Phlebotomy Tourniquets
}

\author{
DONNA M HENSLEY, KEVIN J KRAULAND, DAVID L MCGLASSON
}

OBJECTIVE: A study was undertaken to determine the incidence of Acinetobacter baumannii and methicillin resistant Staphylococcus aureus (MRSA) contamination on reusable phlebotomy tourniquets at Wilford Hall Medical Center, Lackland AFB, TX.

DESIGN: Reusable tourniquets $(\mathrm{n}=200)$ were collected after being used for one day in the outpatient blood collection center $(n=100)$ or during morning blood collection rounds on inpatient wards $(\mathrm{n}=100)$. Tourniquets were cultured and growth was screened for A. baumannii and $S$. aureus. A. baumannii isolates were identified using colonial morphology, oxidase, and GNI+ card on Vitek Legacy. S. aureus isolates were identified and screened for MRSA using colonial morphology, catalase, Staphaurex, and Oxacillin screening agar.

RESULTS: Each outpatient tourniquet was used on an average of 33 patients and each inpatient tourniquet was used on an average of 11 patients. The overall contamination rate was $9 \%(18 / 200)$. A. baumannii was isolated from $11 \%(11 / 100)$ of the outpatient tourniquets and $3 \%(3 / 100)$ of the inpatient tourniquets. Methicillin-susceptible $S$. aureus was isolated from $2 \%(2 / 100)$ of the outpatient tourniquets and $3 \%(3 / 100)$ of the inpatient tourniquets. No MRSA was isolated. One outpatient tourniquet had both $A$. baumannii and methicillin-susceptible $S$. aureus.

CONCLUSIONS: Reusable tourniquets could serve as a potential reservoir for bacterial pathogens.

ABBREVIATIONS: $\mathrm{CDC}=$ Centers for Disease Control; MRSA = methicillin resistant Staphylococcus aureus; TSB = trypticase soy broth; TSA II = trypticase soy agar with $5 \%$ sheep blood; GNI = gram negative identification; BAMC = Brooke Army Medical Center.

INDEX TERMS: MRSA, A. baumannii, tourniquet, infection control

Clin Lab Sci 2010;23(3):151

Donna M. Hensley MT(ASCP), 59 th Clinical Research Division, Lackland AFB, TX 78236-9908.

David L. McGlasson, MS, MLS(ASCP) ${ }^{C M}$, 59th Clinical Research Division, Lackland AFB, TX 78236-9908

Kevin J Krauland, MD, 59 th Laboratory Support Squadron, Lackland AFB, TX 78236-9908

Address for Correspondence: Donna Hensley MT(ASCP), Research Medical Technologist, 59 $9^{\text {th }}$ MDW/SGVUL, 59 th Clinical Research Division, 2200 Bergquist Dr, Bldg 4430, Lackland AFB, TX 78236-9908. (210) 292-6690, (210) 292-2897 (fax), donna.hensley@lackland.af.mil.

\section{INTRODUCTION}

Nosocomial infections represent a significant public health problem, with a high cost in money, morbidity, and mortality. During the 1990s, the Centers for Disease Control (CDC) estimated that, directly or indirectly, the annual number of deaths related to nosocomial infection was 88,000 . The rate of nosocomial infection is estimated at nearly $10 / 100$ patients admitted to the hospital and the estimated annual cost of nosocomial infection with antibioticresistant organisms is up to $\$ 4.5$ billion. ${ }^{1}$ On average, hospital stays for methicillin-resistant Staphylococcus aureus (MRSA) infections cost $\$ 14,000$ compared with 


\section{RESEARCH AND REPORTS}

$\$ 7,600$ for all other stays, and the length of hospitalization was more than double-10.0 days for MRSA infections versus 4.6 days for all other stays. ${ }^{2}$

Common sites of nosocomial infection are the urinary tract, lungs, surgical sites, gastrointestinal tract (particularly with Clostridium difficile) and the blood. The site of a nosocomial infection determines which organisms are most likely to be isolated. Escherichia coli causes most nosocomial urinary tract infections. ${ }^{3}$ Causes of hospital-acquired pneumonia include both grampositive (Streptococcus pneumonia, methicillin resistant and susceptible $S$. aureus) and gram-negative (Haemophilus influenza, Enterobacter spp, Klebsiella penumoniae, E. coli) organisms. ${ }^{4}$ Infections of surgical sites are commonly due to methicillin resistant and susceptible S. aureus, coagulase-negative Staphylococci, Enterococci, and E. coli. ${ }^{5}$

Acinetobacter is a class of gram-negative aerobe that is ubiquitous in soil and water and is also found as normal flora in healthy individuals. Acinetobacter species have been shown to survive for periods of up to a year on a variety of surfaces such as polystyrene, ceramic, polyvinyl chloride, stainless steel, and rubber. They are an important cause of nosocomial infections, causing pneumonia, burn wound infection, urinary tract infection, sepsis, and endocarditis. ${ }^{6-11}$ A. baumannii is particularly important among the pathogenic Acinetobacter species as it is characterized by a high level of antimicrobial resistance and is displaying increasing rates of resistance. ${ }^{12}$ Resistance to meropenem has increased from $5.9 \%$ in 1998 to $28.6 \%$ in $2005^{12}$ and Doi et al reported a patient infected with $A$. baumannii that lacked susceptibility to all commercially available antimicrobial drugs. ${ }^{13}$ These qualities, the ability to survive on a variety of surfaces and a high level of antimicrobial resistance, have lead to a growing concern for A. baumannii as a potential agent for hospital acquired infections.

An increase in A.baumannii infections among patients at military medical facilities treating U.S. service members who have been injured in Iraq and Afghanistan has been reported in the literature. Two medical centers, Landstuhl Regional Medical Center and Walter Reed Army Medical Center, identified 102 patients with blood cultures positive for A. baumannii during the period January 1, 2002 - August 31, 2004. The two medical centers had a combined total of three cases of $A$. baumannii positive blood culture during the previous two years. ${ }^{14}$ Brooke Army Medical Center (BAMC) reported twenty-three soldiers wounded in Iraq and subsequently admitted to BAMC who had wounds that were culture positive for $A$. baumannii during the period of March 2003 to May 2004. Eighteen of the twenty three patients had osteomyelitis which had not been identified at BAMC during the fourteen months preceding March 2003. ${ }^{15}$

Studies involving environmental and colonization cultures indicate that the source of the A. baumannii infections is nosocomial in origin. A study conducted at BAMC that included 293 soldiers with no history of deployment and who were not healthcare workers found no Acinetobacter nares colonization in any of the participants indicating that $A$. baumannii nares colonization in a normal healthy population is very low. ${ }^{16} \mathrm{~A}$ study assessing the bacteriology of war wounds at the time of injury sampled 61 separate acute traumatic injury wounds from 49 casualties upon arrival at the $31^{\text {st }}$ Combat Support Hospital in Baghdad. The study revealed a predominance of gram positive organisms of low virulence and pathogenicity. No multiple drug resistant gram negative organisms were recovered. ${ }^{17}$ Another study, conducted in Iraq and Kuwait by the Walter Reed Army Institute of Research, found skin colonization in only 1 of 160 patients who were screened and in only 1 of 49 soil samples but $A$. baumannii-calcoaceticus complex isolates were recovered from treatment areas in all 7 of 7 field hospitals sampled. ${ }^{18}$ A study at BAMC determined the antimicrobial susceptibilities of $142 \mathrm{~A}$. baumanniicalcoaceticus complex isolates, 95 of which were from wounded U.S. soldiers deployed overseas, and found broad antimicrobial resistance among the isolates tested. The isolates from deployed patients were more resistant than the isolates from nondeployed patients though the reason for this is unclear. ${ }^{19}$

A. baumannii continues to pose a serious infection control problem in military healthcare settings and has been implicated in one case of occupational transmission from a U.S. serviceman wounded in Iraq to a health care worker. ${ }^{20-23}$ 


\section{RESEARCH AND REPORTS}

Previous studies have established that MRSA can also survive for days to months on environmental surfaces, including stethoscopes, tabletops, patient charts, and tourniquets. ${ }^{24-32}$

Instrumentation procedures involving sterile sites may provide a point of entry for pathogenic organisms. Additionally, pathogens may be spread from patient to patient by contaminated environmental surfaces, equipment, or the hands of healthcare workers. ${ }^{1}$ Some of these surfaces, such as beds, tables, hygroscopic bandages, curtains, and infusion pumps, have been implicated as reservoirs for transmission of disease in hospital settings. ${ }^{11,33-41}$

Studies reported in the literature have shown that, while not implicitly implicated in the spread of transmissible disease, reusable tourniquets may serve as a possible bacterial reservoir. ${ }^{26,31,32,41,42}$ Total $S$. aureus contamination rates on the tourniquets examined in the studies cited ranged from 5\%(10/200) to $77.8 \%$ (28/36) 26,31,39,40,41 and contamination rates for MRSA specifically ranged from 0 to $41.7 \%(15 / 36))^{26,31,32,39,40,41}$ In one study Acinetobacter spp was included on the list of skin flora isolated from reusable tourniquets but the rate of recovery was not given. ${ }^{39}$ To our knowledge there are no reports in the literature on the subject of $A$. baumannii contamination rates on reusable tourniquets.

The length of time that the tourniquets in the cited studies had been in use varied from one day to 4 years. ${ }^{26,31,32,40}$ Leitch et al supplied fresh tourniquets and collected them for evaluation after one day of use. They found an MRSA contamination rate of $24.4 \%(32 / 131)$ indicating that daily replacement will not eliminate the potential bacterial reservoir. ${ }^{26}$ Sacar et al collected tourniquets $(n=36)$ before and after implementation of hospital infection control measures that included an educational program to health care personnel by members of the infection control program that reemphasized infection control measures, repeated every 3 months, and a visual display with color posters that emphasized the importance of handwashing. They found a $36.1 \%$ decrease $(41.7 \%$ to $5.6 \%)$ in the number of tourniquets contaminated with MRSA one year after implementation of the new procedures, demonstrating the effectiveness of infection control procedures in decreasing but not eliminating the rate of contamination. ${ }^{41}$ These studies indicate that a combination of actions would be needed to eliminate tourniquets as a potential source of bacterial infection.

Our study examined MRSA and A. baumannii contamination rates on reusable tourniquets used in outpatient and inpatient settings in a Department of Defense tertiary care hospital.

\section{METHODS}

Reusable tourniquets (Fisher HealthCare, Houston, TX) were collected after being used for one day in the outpatient blood collection center $(n=100)$ or during morning blood collection rounds on inpatient wards $(\mathrm{n}=100)$. The used tourniquets were placed in sterile specimen collection bags (VWR International, Suwanee, GA) and transported to the lab. Unused tourniquets $(\mathrm{n}=10)$ were included as controls. Tourniquets were transferred to sterile specimen cups, covered with $80 \mathrm{~mL}$ trypticase soy broth (TSB) (Becton, Dickinson and Company, Sparks, MD) and incubated at $35 \pm 2^{\circ} \mathrm{C}$. After overnight incubation the TSBs were subcultured to trypticase soy agar with 5\% sheep blood (TSAII, BBL/BD, Sparks, MD) and MacConkey agar (BBL/BD, Sparks, MD). Plates were examined after $18-24$ hours incubation at $35 \pm 2^{\circ} \mathrm{C}$ in ambient air. The detection limit for both $S$. aureus and A. baumannii using this enrichment procedure with disposable tourniquets was determined by serial dilution to be $10^{4} \mathrm{CFU} / \mathrm{mL}$. A. baumannii isolates were identified using colonial morphology, negative oxidase reaction, identification of $A$. calcoaceticus-baumannii complex using the GNI+ card on a Vitek Legacy instrument (bioMerieux, Durham, NC), and non hemolytic reaction on TSA II. S. aureus isolates were identified using colonial morphology, positive catalase reaction (BD, Sparks, MD), and positive Staphaurex reaction (Remel, Lenexa, KS). Methicillin resistance was determined by inoculating $S$. aureus isolates to Oxacillin screening agar (BBL/BD, Sparks, MD). Growth on the Oxacillin screening agar was interpreted as methicillin resistance. No growth on the Oxacillin screening agar was interpreted as methicillin susceptibility. No susceptibility testing was performed on the $A$. calcoaceticus-baumannii complex isolates. 


\section{RESEARCH AND REPORTS}

\section{RESULTS}

Each outpatient tourniquet was used on an average of 33 patients and each inpatient tourniquet was used on an average of 11 patients. The overall contamination rate was 9\% (18/200). A. baumannii was isolated from $11 \%(11 / 100)$ of the outpatient tourniquets and $3 \%$ $(3 / 100)$ of the inpatient tourniquets. Methicillinsusceptible $S$. aureus was isolated from $2 \%(2 / 100)$ of the outpatient tourniquets and $3 \%(3 / 100)$ of the inpatient tourniquets. One outpatient tourniquet had both $A$. baumannii and $S$. aureus. No MRSA was isolated. A. baumannii and $S$. aureus were not isolated from any of the control tourniquets.

\section{DISCUSSION}

Acinetobacter species are capable of survival on environmental surfaces for months and this creates a potential reservoir for infection. ${ }^{24,37}$ The incidence of $A$. baumannii contamination of reusable tourniquets has not previously been studied. Given the problems that $A$. baumannii has caused in military healthcare facilities since the Gulf War we decided to look at the incidence of $A$. baumannii on the reusable tourniquets in our facility which is a Department of Defense tertiary care hospital.

A. baumannii has a reputation as a hospital-associated pathogen, so we were surprised that the rate of contamination with $A$. baumannii was so much higher on outpatient than inpatient tourniquets $(11.0 \%$ vs $3.0 \%)$. There are several possible reasons for this. It could be that, on average, the outpatient tourniquets were used on more patients than the inpatient tourniquets (33 vs. 11) thus increasing the opportunity for contamination. Another explanation could be that our outpatient population has a higher rate of colonization with $A$. baumannii than our inpatient population but this is unlikely since the inpatient population is drawn from the outpatients. It is also possible that one or more of the phlebotomists working in the outpatient drawing facility is colonized with $A$. baumannii or that other environmental surfaces in the outpatient drawing facility are contaminated with $A$. baumannii resulting in spread of the $A$. baumannii to the tourniquets. For the purposes of this study we did not attempt to rule out any of these possibilities.
While Acinetobacter is most commonly thought of as a causative agent of hospital-acquired pneumonia, it is also known to cause community-acquired pneumonia. It is possible that these outpatient tourniquets represent a potential vector for community-acquired $A$. baumannii infection. One retrospective study of $A$. baumannii patients found that those with communityacquired disease had a higher 30 day mortality rate than those with hospital-acquired disease (57.8\% vs. $35.4 \%)$. However, these patients were also significantly more likely to have ever smoked and to have had chronic obstructive pulmonary disease. ${ }^{42}$ It may be that the same underlying conditions predisposing these patients to develop community-acquired disease also herald a poor prognosis. Whatever the underlying conditions this type of study reinforces the significance of community acquired disease in the infectious disease arena.

It was also surprising that in our setting there was no evidence of MRSA contamination on the tourniquets after approximately 4400 subject contacts by the phlebotomists. It is possible that the phlebotomists and infection control programs are more aware of MRSA and take measures such as dedicated tourniquets for known MRSA patients to prevent the spread of MRSA on multi use items such as tourniquets. We feel that the lack of MRSA contamination is the result of an effective infection control program and perhaps the program needs to be broadened to include all potential multi drug resistant pathogens instead of focusing on only the best known ones.

All of our tourniquets were replaced on a daily basis for the study and we collected the tourniquets at the end of the day. However, if a tourniquet was contaminated with blood during the process of a venipuncture procedure the tourniquet was disposed of and another was obtained for use. We did not culture any blood contaminated tourniquets nor did we evaluate how often the phlebotomists replaced their tourniquets during the day. Differences in practices with regard to hygiene and infection control as well as the efficacy of sanitation control protocols are other possible contributing factors which we did not attempt to evaluate or control in this study. In addition we did not attempt to identify any differences in the inpatient and 


\section{RESEARCH AND REPORTS}

outpatient isolates or determine the antimicrobial susceptibility patterns.

We were encouraged by the low rate of contamination of $A$. baumannii and MRSA on the tourniquets used by the phlebotomy teams in our hospital. However, any rate of contamination is cause for concern. It is likely that a combination of actions would be necessary to eliminate phlebotomy tourniquets as a potential reservoir for nosocomial infections. Even instituting a policy for single use tourniquets would probably not be sufficient since a phlebotomist with poor hand hygiene could transfer pathogens from the last patient to the new tourniquet. Infection control is a constantly evolving process and we must be vigilant in anticipating the need for and implementing the improved methods necessary to provide a safe environment for our patients.

\section{DISCLAIMER}

This material represents the personal statements of the authors and is not intended to constitute an endorsement by the $59^{\text {th }}$ Medical Wing or any other federal entity.

\section{ACKNOWLEDGEMENTS}

This research was performed under the authority of the Department of Defense and the $59^{\text {th }}$ Medical Wing, $59^{\text {th }}$ Clinical Research Division, Lackland AFB, TX, Institutional Review Board.

\section{REFERENCES}

1. Henderson DK, Fishman N. "Chapter 304: Prevention and control of hospital-acquired infections." Goldman L, editor. Cecil Medicine. 23rd ed. Philadelphia: Saunders Elsevier; 2007.

2. Elixhauser A, Steiner C. Statistical Brief \#35, Infections with methicillin-resistant Staphylococcus aureus (MRSA) in U.S. hospitals, 1993-2005. July 2007. http://www.hcupus.ahrq.gov/reports/statbriefs/sb35.jsp

3. Schaeffer A, Schaeffer E. "Chapter 8: Infections of the urinary tract." Wein AJ, editor. Campbell-Walsh Urology. 9th ed. Philadelphia: Saunders Elsevier; 2007.

4. Macfarlane JT, Baldwin DR. "Chapter 35: Hospital-acquired pneumonia.” Cohen J, Powderly WG, Berkley SG et al, editors. Infectious Diseases. 2nd ed. Philadelphia: Mosby; 2004.

5. Anaya DA, Dellinger EP. "Chapter 14: Surgical infections and choice of antibiotics." Townsend CM, Beauchamp RD, Evers BM et al., editors. Sabiston Textbook of Surgery. 18th ed. Philadelphia: Saunders Elsevier; 2007.
6. Bergogne-Bérézin E. "Chapter 229: Pseudomonads and miscellaneous gram-negative bacilli." Cohen J, Powderly WG, Berkley SG et al., editors. Infectious Diseases. 2nd ed. Philadelphia: Mosby; 2004.

7. Lee HW, Koh YM, Kim J et al. Capacity of multidrug-resistant clinical isolates of Acinetobacter baumannii to form biofilm and adhere to epithelial cell surfaces. Clin Microbiol Infect 2008;14(1):49-54.

8. Wendt C, Dietze B, Dietz E, Ruden H. Survival of Acinetobacter baumannii on dry surfaces. J Clin Micro 1997;35(6):1394-7.

9. Jawad A, Seifert H, Snelling AM et al. Survival of Acinetobacter baumannii on dry surfaces: comparison of outbreak and sporadic isolates. J Clin Micro 1998;36(7):1938-41.

10. Jawad A, Heritage J, Snelling AM et al. Influence of relative humidity and suspending menstrua on survival of Acinetobacter spp. on dry surfaces. J Clin Micro 1996;34(12):2881-7.

11. Catalano M, Quelle LS, Jeric PE et al. Survival of Acinetobacter baumannii on bed rails during an outbreak and during sporadic cases. J Hosp Inf 1999; 42:27-35.

12. Perez F, Hujer AM, Hujer KM et al. Global challenge of multidrug-resistant Acinetobacter baumannii. Antimicrob Agents Chemother 2007;370:2030-43.

13. Doi Y, Husain S, Potoski BA et al. Extensively drug-resistant Acinetobacter baumannii. Emerg Infect Dis 2009;15(6):980-2.

14. Centers for Disease Control and Preventions. 2004. Acinetobacter baumannii infections among patients at military medical facilities treating injured US service members, $2002-$ 2004. MMWR Morb Mortal Wkly Rep; 53:1063-6.

15. Davis KA, Moran KA, McAllister CK et al. Multi-drug resistant Acinetobacter extremity infections in soldiers. Emerg Infec Dis 2005;11(8):1218-24.

16. Griffith ME, Ellis MW, and Murray CK. Acinetobacter nares colonization of healthy US soldiers. Infect Cont and Hosp Epidemiol 2006;27(7):787-8.

17. Murray CK, Roop SA, Hospenthal DR et al. Bacteriology of war wounds at the time of injury. Military Med 2006;171(9):826-9.

18. Scott PG, Deye A, Srinivasan C et al. An outbreak of multidrug-resistant Acinetobacter baumannii-calcoaceticus complex infection in the US military health care system associated with military operations in Iraq. Clin Inf Dis 2007;44(12): 1577-84.

19. Hawley J.S, Murray CK, Griffith ME et al. Susceptibility of Acinetobacter strains isolated from deployed U.S. military personnel. Antimicrob Agents and Chemo 2007; 51(1):376-8.

20. Whitman $T$. Infection control challenges related to war wound infections in the ICU setting. J Trauma 2007;62(6):S53.

21. Petersen K, Riddle MS, Danko JR et al. Trauma-related infections in battlefield casualties from Iraq. Annals of Surgery 2007;245(5):803-11.

22. Colombo CJ, Mount CA, Popa CA. Critical care medicine at Walter Reed Army Medical Center in support of the global war on terrorism. Crit Care Med 2008;36(7):S388-94.

23. Whitman TJ, Qasba SS, Timpone JG et al. Occupational transmission of Acinetobacter baumannii from a United States 


\section{RESEARCH AND REPORTS}

serviceman wounded in Iraq to a health care worker. Clin Infect Disease 2008;47(4):439-43.

24. Kramer A, Schwebke I, Kampf G. How long do nosocomial pathogens persist on inanimate surfaces? A systematic review. BMC Infect Dis 2006;6:130.

25. Huang R, Mehta S, Weed D et al. Methicillin-resistant Staphylococcus aureus survival on hospital fomites. Infect Control Hosp Epidemiol 2006;27:1267-9.

26. Leitch A, McCormick I, Gunn I et al. Reducing the potential for phlebotomy tourniquets to act as a reservoir for methicillinresistant Staphylococcus aureus. J of Hosp Infect 2006;63:42831.

27. Dietze B, Rath A, Wendt C, Martiny H. Survival of MRSA on sterile goods packaging. J Hosp Infect 2001;49(4):255-61.

28. Marinella MA, Pierson C, Chenoweth C. The stethoscope: A potential source of nosocomial infection? Arch Internal Med 1997;157:786-90.

29. Merlin MA, Wong ML, Pryor PW et al. Prevalence of methicillin-resistant Staphylococcus aureus on the stethoscopes of emergency medical services providers. Prehospital Emergency Care 2009;13:71-4.

30. Williams C, Davis DL. Methicillin-resistant Staphylococcus aureus fomite survival. Clin Lab Sci 2009;22(1):34.

31. Franklin GF, Bal AM, McKenzie H. Phlebotomy tourniquets and MRSA. J Hosp Infect 2007;65:173-84.

32. Berman DS, Schaefler S, Simberkogg MS, Rahal JJ. Tourniquets and nosocomial methicillin-resistant Staphylococcus aureus infections. NE J of Med 1986;315(8):514-5.

33. Aygun G, Demirkiran O, Utku $\mathrm{T}$ et al. Environmental contamination during a Carbapenem-resistant Acinetobacter baumannii outbreak in an intensive care unit. J Hosp Inf 2002;52:259-62.
34. Wang SH, Sheng WH, Chang YY et al. Healthcare-associated outbreak due to pan-drug resistant Acinetobacter baumannii in a surgical intensive care unit. J Hosp Infect 2003;53(2):97-102.

35. Denton M, Wilcox MH, Parnell P et al. Role of environmental cleaning in controlling an outbreak of Acinetobacter baumannii on a neurosurgical intensive care unit. J Hosp Infect 2004;56(2):106-10.

36. Melamed R, Greenberg D, Porat N et al. Successful control of an Acinetobacter baumannii outbreak in a neonatal intensive care unit. J Hosp Infect 2003;53(1):31-8.

37. Das I, Lambert P, Hill D et al. Carbapenem-resistant Acinetobacter and role of curtains in an outbreak in intensive care units. J of Hosp Infect 2002;50:110-4.

38. Forseter G, Joline C, Wormser GP. Blood contamination of tourniquets used in routine phlebotomy. Amer J Inf Control 1990;18(6):386-90.

39. Golder M, Chan CLH, O'Shea S et al. Potential risk of crossinfection during peripheral-venous access by contamination of tourniquets. Lancet 2000;355:44.

40. Rourke C, Bates C, Read RC. Poor hospital infection control practice in venipuncture and use of tourniquets. J Hosp Inf 2001;49(1):59-61.

41. Sacar S, Turgut H, Kaleli I et al. Poor hospital infection control practice in hand hygiene, glove utilization, and usage of tourniquets. Amer J Inf Control 2006;34(9):606-9.

42. Leung WS, Chu CM, Tsang KY et al. Fulminant communityacquired Acinetobacter baumannii pneumonia as a distinct clinical syndrome. Chest 2006;129(1): 